\title{
Salidas terapéuticas: espacio lúdico para la integración
}

RESUMEN: Las actividades de ocio y tiempo libre deben ser una parte importante de cualquier programa integral de rehabilitación en personas con enfermedad mental grave.

PALABRAS CLAVE: monitoras de rehabilitación psicosocial, ocio, autoestima, integración social.
SUMMARY: A Program for preparation for leisure time it seems to us a esencial element of a Rehabilitation Unit for patients with an important mental ilness.

KEY WORDS: manager of psychosocial rehabilitation, leisure time, selfcenteredness, social integration.

\section{Introducción}

Hoy en día, en pleno siglo XXI, el tiempo de ocio del que disponemos es cada vez mayor, y el modo de su disfrute constituye un importante indicador de la calidad de vida en las actuales sociedades occidentales. Por una parte, es considerado como un espacio donde el ejercicio de las libertades individuales se hace más patente. La persona decide y gestiona qué actividades quiere realizar en función de sus intereses (descanso, diversión, compañía, actividades culturales, etc.). Por otra parte, las actividades de ocio pueden proporcionar un alto grado de satisfacción y realización personal.

Por estas razones, no podemos obviar que las actividades de ocio y tiempo libre deben ser una parte importante de cualquier programa integral de rehabilitación en personas con enfermedad mental severa. Estas personas, por lo general, disponen de más tiempo libre con el que no saben qué hacer y el aprender a gestionarlo puede suponer una oportunidad de integración social y comunitaria. De todas formas, podemos hacer un repaso de los beneficios de las actividades de ocio siguiendo a Cañamares y cols. (2001):

a) Facilitan que el paciente recupere un papel activo y protagonista en ciertos aspectos de su vida. Como hemos comentado antes, el tiempo de ocio es una de las facetas donde se hace más patente el ejercicio de las libertades individuales.

b) Dotan de sentido para el paciente algunos entrenamientos que antes rechazaba. Es importante saber expresarse para comprar una entrada de cine o pedir algo en un bar; es importante ir aseado a los sitios, etc.

c) Mejoran las expectativas de los pacientes respecto de su recuperación, ya que relativizan el impacto negativo que la enfermedad tiene en sus vidas.

d) Permite evaluar y entrenar múltiples capacidades de los pacientes: normalmente al ser actividades de alta motivación se crean unas condiciones privilegiadas para el aprendizaje con refuerzo inmediato. Por otro lado, el descubrir qué actividades resultan satisfactorias para un paciente puede servir como refuerzo de otras que le cuesten más o en las que tenga más dificultades. 
e) Facilita el desarrollo de una buena relación terapéutica con todos los profesionales.

f) Como el grado de satisfacción aumenta con las actividades de ocio a la vez que hace que disminuya la ansiedad y la irritabilidad, esto necesariamente tendría que traducirse en una disminución de la sintomatología negativa y en un mejor manejo de la positiva.

En la Unidad Comunitaria de Rehabilitación (U.C.R.) Son Gibert (Palma de Mallorca), conscientes de la importancia que el buen manejo del ocio y del tiempo libre tienen para la rehabilitación e integración comunitaria, se ha puesto en marcha un programa específico de ocio y tiempo libre.

Esta Unidad, como tal, en la Comunidad (pues procede de la transformación de otra Unidad encargada de la desinstitucionalización desde el Hospital Psiquiátrico) lleva funcionando desde abril de 2003. En ella trabaja un equipo multi-interdisciplinar compuesto por una psiquiatra (coordinadora del Centro), un trabajador social, una psicóloga clínica, un terapeuta ocupacional, una enfermera de Salud Mental, un monitor administrativo y cuatro monitoras de rehabilitación psicosocial. Se llevan a cabo diferentes programas que abarcan diversas áreas de intervención para dar un abordaje integral desde una perspectiva biopsicosocial.

Más en concreto, el programa de ocio y tiempo libre es llevado a cabo fundamentalmente por las monitoras de rehabilitación psicosocial. El diseño del programa se realiza siguiendo tanto la línea del resto de actividades realizadas en el centro, reforzando los aprendizajes realizados a través de otros programas, como (y muy importante) los objetivos marcados en el Plan Individual de Tratamiento de cada usuario.

El programa de ocio está destinado prácticamente a todos los usuarios que acuden a esta UCR. Los objetivos concretos del programa, además de los expuestos más arriba, son:

- Fomentar la participación en las actividades del programa de ocio y tiempo libre.

- Convertir el tiempo de este espacio en una ocupación agradable y de disfrute.

- Evitar el aislamiento.

- Fomentar las relaciones interpersonales.

- Fomentar la tolerancia.

- Fomentar el respeto.

- Entrenar y modelar in situ el manejo del transporte público, circulación vial, orientación dentro del núcleo urbano, manejo de planos, manejo de imprevistos, manejo de «dinero de bolsillo». 
- Aprender a utilizar la información adecuadamente.

- Fomentar el interés por la cultura popular.

- Fomentar el interés por la cultura.

- Fomentar la puntualidad.

Para lograr estos objetivos se eligen actividades que van dirigidas a:

- Conocer nuestra isla y ciudad.

- Conocer nuestra cultura.

- Aprender a cuidar nuestro entorno.

- Participar en talleres lúdicos.

- Participar en talleres de arbitraje y consumo.

- Visitas a exposiciones.

- Visitas a museos.

- Visitas guiadas por la ciudad.

- Paseos por la ciudad.

- Comidas de celebración en restaurantes.

- Hábitos saludables de consumo y uso adecuado del «dinero de bolsillo» (consumiciones en locales de la zona).

Para llevar a término las actividades se está en permanente contacto tanto con entidades públicas como con las privadas.

Asimismo, se sigue trabajando lo realizado en las salidas desde el Taller de Revista de la U.C.R., donde los usuarios realizan un breve resumen de las salidas, recogen impresiones o experiencias de la misma. El coste de las salidas suele ser gratuito a través de subvenciones; si éstas no son posibles, los gastos los asume la UCR, al igual que los desplazamientos fuera de la ciudad, excepto los pequeños gastos antes mencionados, que son utilizados como herramienta de trabajo terapéutico.

El grupo Delfín. Dado que el objetivo final de la rehabilitación (como de cualquier otro tratamiento psicoterapéutico) es el logro de la máxima autonomía con la puesta en marcha de los recursos personales apropiados a cada momento, desde la UCR se ha puesto en marcha un subprograma dentro del programa de ocio y tiempo libre. Este subprograma está destinado a un grupo más reducido de usuarios (entre 8 y 10). La característica diferencial está en que las salidas son realizadas SIN el acompañamiento de las monitoras. Los objetivos marcados son los mismos así como las actividades a realizar. A esto se añade el aprender a responsabilizarse de la actividad desde el inicio hasta el final, el lograr una cohesión de grupo y el poner en marcha sus propios recursos logrando así el máximo de autonomía y «normalización» en pasos sucesivos. 
La monitora de la UCR encargada del programa tiene contacto directo, antes y después, con la entidad que van a visitar los usuarios, para poder recoger información de la salida, esto es, puntualidad, atención, uso adecuado de las instalaciones, etc.

A los usuarios previamente se les posibilita la búsqueda de información sobre el local o lugar que visitarán, de lo que van a ver, historia, etc. Además de una persona de contacto, medio de transporte a utilizar, orientación del punto de encuentro, hora de encuentro, horario de la visita. Cada día se asigna a un usuario para centralizar toda la información y posibles incidencias, con lo que se fomenta el sentimiento de responsabilidad personal y la solidaridad con el grupo.

Muestra: desde abril de 2003 hasta abril de 2005 han pasado por la UCR Son Gibert 49 usuarios que, al menos, han realizado una salida terapéutica. De éstos, 32 son varones y 17 mujeres. Su rango de edad está entre los 20 años y los 55 (media $=37,7$; desviación estándar $=8,9$ ).

En cuanto al diagnóstico principal, según criterios DSM-IV, 19 usuarios estaban diagnosticados de esquizofrenia paranoide; 8 de esquizofrenia indiferenciada; 3 de esquizofrenia desorganizada; 5 de esquizofrenia residual; 5 de trastorno psicótico inespecífico; 6 de trastorno esquizoafectivo; 1 de trastorno bipolar y 2 de trastorno límite de personalidad.

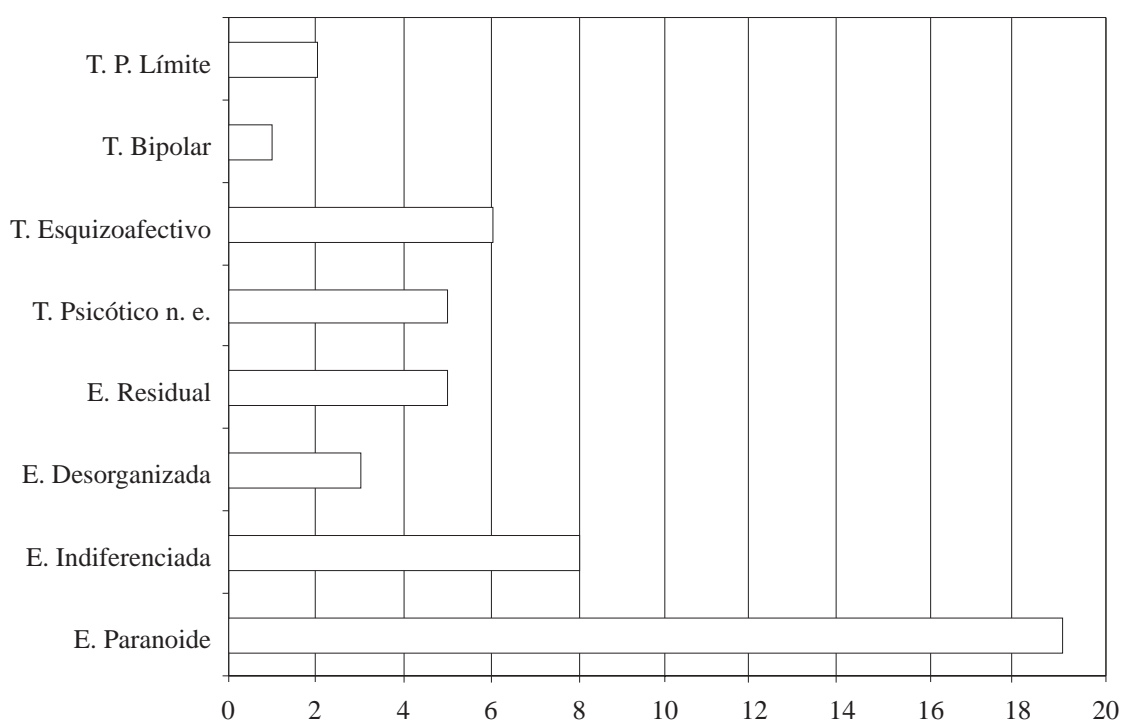


Proceso-resultados: en estos dos años se han contabilizado 100 salidas realizadas por parte del grupo menos autónomo, que las podríamos categorizar en:

- Salidas a exposiciones en lugares cerrados: 39.

- Visitas culturales (paseos, actividades de interés científico y de conocimiento del entorno): 31 .

- Salidas nocturnas («cinema a la fresca», paseo, feria, etc.): 8.

- Salidas a la playa (estamos en Mallorca): 14.

- Otras: 8.

Gráfico 2

Tipo de salidas terapéuticas

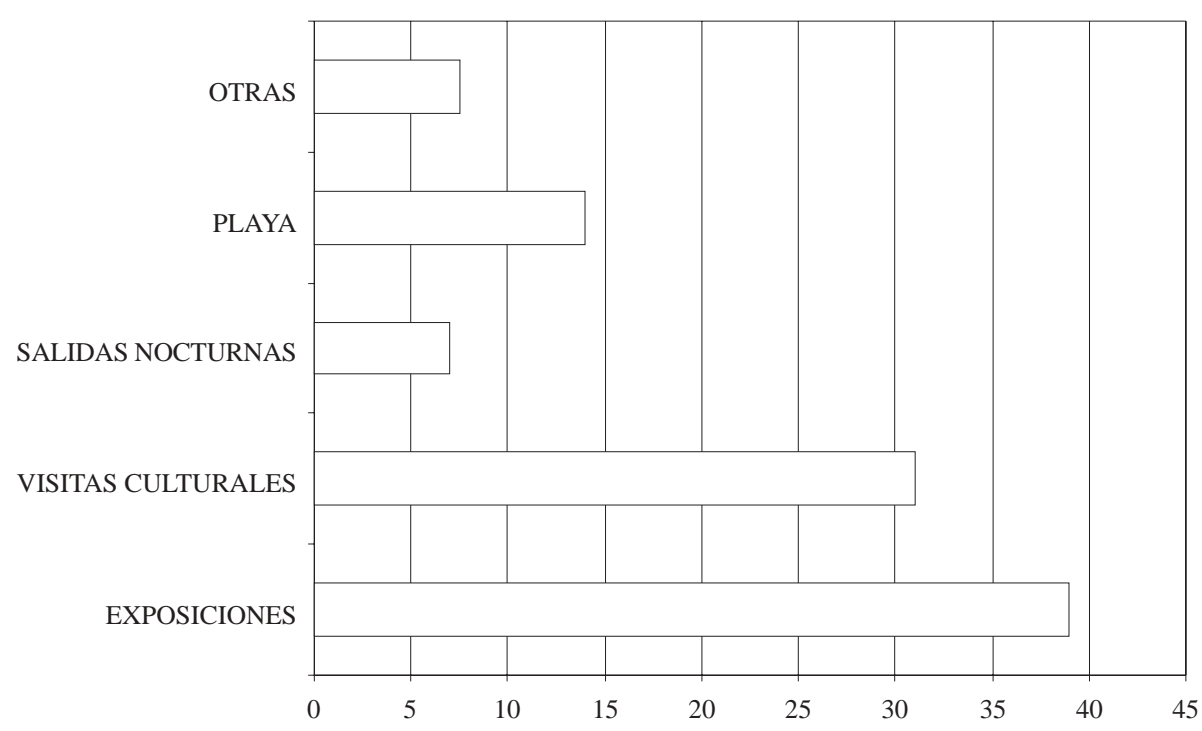

El «grupo Delfín» (grupo más autónomo) por su parte, en este periodo de tiempo, dado que comenzó su puesta en marcha más tarde, ha realizado las siguientes salidas: 7 de carácter cultural, 8 de tipo lúdico, 3 actividades relacionadas con el teatro y 2 relacionadas con el conocimiento del entorno: en total, 20. La media de participación de usuarios ha sido de 6 por salida, lógicamente menos que en el grupo grande para favorecer la autonomía y la integración comunitaria de los participantes. 
Gráfico 3

Grupo Delfín

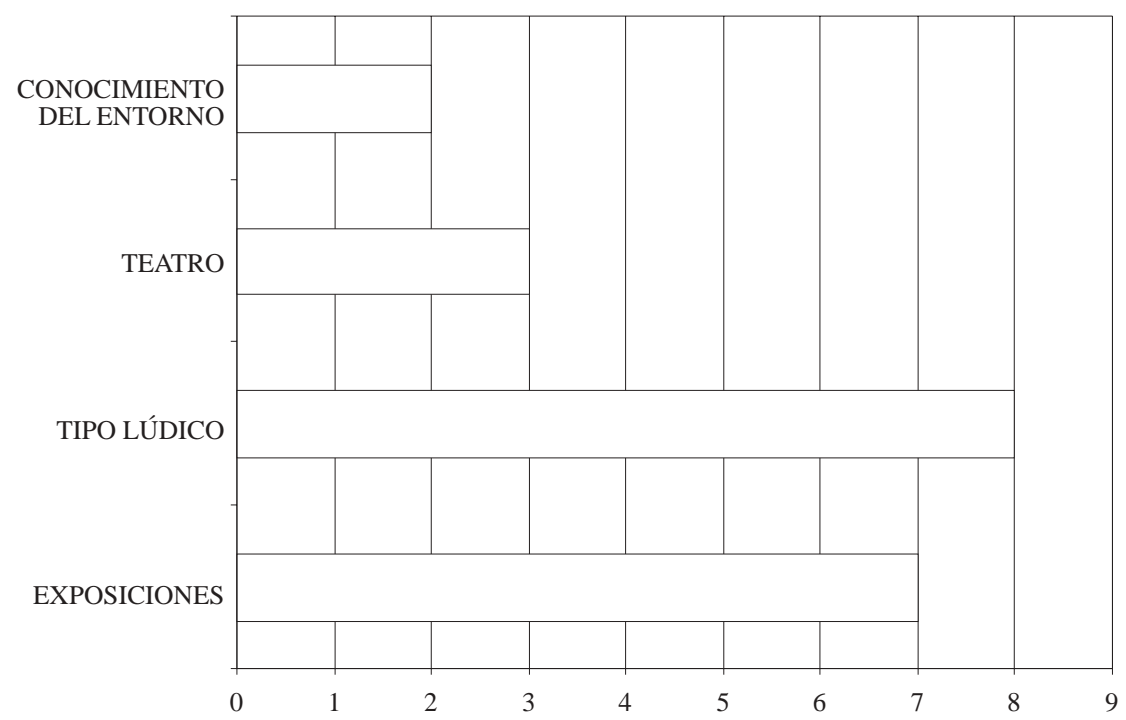

A esto hay que añadir que se han realizado dos viajes: uno a Ibiza y otro a Formentera de tres días de duración cada uno con el número de los usuarios que en ese momento acudía a la UCR. La media de participación en las salidas terapéuticas está entre 15 y 20 usuarios, y son acompañados por tres monitoras. Esto hace que haya una ratio profesional/usuarios adecuada para poder trabajar los diferentes aspectos de la rehabilitación. Entre éstos, destacamos:

- Actividades de la vida diaria: aseo, aspecto y vestido adecuados, tanto al entorno como a la actividad a realizar.

- Habilidades sociales: mejora de la forma de relación con los demás; hacer peticiones adecuadamente, solicitar información, no interrumpir a los guías de las visitas.

- Manejo del dinero: traer el dinero suficiente para cuando hace falta, y que no sea gastado antes de tiempo.

- Puntualidad.

- Utilización de diferentes medios de transporte y de la tarjeta.

- Autoestima e interés por los demás y el entorno. 


\section{Conclusiones}

Desde nuestra experiencia nos gustaría destacar una serie de logros en los usuarios. En esta actividad, los usuarios han ido demostrando un interés progresivo por el disfrute del tiempo libre. Aumenta su autosatisfacción, el grado de independencia y su autoestima. Se han incrementado y mejorado las relaciones interpersonales de los usuarios. Los usuarios demuestran más cuidado en su aspecto personal (aseo, vestimenta, etc.). Ha aumentado su interés por lo que les rodea y su motivación para tener un papel más activo dentro de la sociedad. Aumenta su calidad de vida. Es además un espacio que favorece la desestigmatización de las personas con enfermedad mental crónica, y ayuda a desmontar las ideas erróneas y prejuicios sobre la enfermedad mental. Es un espacio para reunirse y evitar el aislamiento, haciendo actividades conjuntas para asumir y repartir responsabilidades y compromisos.

En definitiva, un espacio que ayuda a mantener a las personas con enfermedad mental grave en la Comunidad y de manera autónoma.

Nuestra intención, desde la UCR Son Gibert y desde el grupo de monitoras de rehabilitación de esta Unidad es seguir trabajando por la autonomía e integración social de estas personas; siendo este espacio de las salidas terapéuticas un lugar privilegiado. Parafraseando a Cavafis diremos que no importa donde se llega en las (salidas) sino el camino que se recorre.

\section{BIBLIOGRAFÍA:}

(1) AA.VV., Cuaderno técnico $n .^{\circ}$ 6: Rehabilitación psicosocial del trastorno mental severo. Situación actual y recomendaciones, Madrid, AEN, 2002.

(2) Aznar, E.; Berlanga, A., Guía práctica para el manejo de la esquizofrenia: manual para la familia y el cuidador, Madrid, Pirámide, 2004.

(3) CAÑAmares, J. M. (comp.), Esquizofrenia, Madrid, Síntesis, 2001.

(4) GISBERT, C. (coord.), Rehabilitación psicosocial y tratamiento integral del Trastorno mental severo, Madrid, AEN, 2003.

(5) RodrígueZ, A. (comps), Rehabilitación psicosocial de personas con Trastornos mentales crónicos, Madrid, Pirámide, 1997.

* Francisca Tur Juan; M. ${ }^{a}$ Jesús Armada; Deo González Aguilar; Ana M. ${ }^{a}$ Segura Valero. Monitoras de rehabilitación psicosocial. Unidad Comunitaria de rehabilitación (U.C.R), Palma de Mallorca (Baleares). Unidad Comunitaria de Rehabilitación de Son Gibert.

Correspondencia: Francisca Tur Juan. UCR Son Gibert. Passeig de Marratxí n. ${ }^{\circ}$ 1, bajos. 07008 Palma de Mallorca (Baleares). lauraftered@ hotmail.com 